\title{
THE SIGNIFICANCE OF THE FINE STRUCTURE OF ARTICULAR CARTILAGE
}

\author{
Peter Bullough, New York, U.S.A., and John Goodfellow, Oxford, England \\ From the Nuffield Department of Orthopaedic Surgery, University of Oxford, \\ and the Nuffield Orthopaedic Centre
}

It is a general rule that the fine structure of a connective tissue is closely related to the forces it transmits. This rule, at its simplest, is exemplified in the longitudinal arrangement of tendon fibres, at its most complex in the trabecular structure of bone. Because fibre bundles in a fascia can be seen with the naked eye and because bone trabeculae are visible in a radiograph we are accustomed to relate the microstructure of these connective tissues both to the gross anatomy and to the forces of compression and tension which they transmit. The interdependence of form and function is no less absolute in articular cartilage, but the fine structure of this material is more difficult to demonstrate. Sections of articular cartilage viewed by simple direct light microscopy reveal a formless glassy or so-called hyaline (Greek iuidevos) matrix, and differential staining methods add little to the picture. It needs a polarising microscope to reveal the pattern of collagen fibres embedded in the ground substance and an electron microscope to demonstrate the individual collagen fibrils. The significance of the fine structure thus revealed can only be understood when it is seen as part of the gross structure.

This paper attempts to correlate the features of healthy and degenerate articular cartilage visible to the naked eye with the microscopic structure of cartilage, of which they are the consequence.

\section{CHONDROMALACIA}

This term is used widely and imprecisely to describe degeneration of cartilage of many degrees of severity. We prefer to restrict its use to the literal meaning-soft cartilage. Such a change is tangible, not visible, and the surface is smooth and intact.

The apparent stiffness of articular cartilage will of course vary with its thickness, but even when this variation is allowed for it has been shown that there are considerable variations in the real stiffness of the cartilage over an articular surface (Kempson 1967). In 1877 Weichselbaum commented upon the softness of the perifoveal cartilage as compared to that covering other parts of the femoral head. Recently Kempson (1967) has shown that on the femoral head there are contours of cartilage stiffness in a pattern which is consistent from subject to subject and from youth to old age. The present authors have described areas of soft cartilage and correlated these changes to the pattern of load distribution (Goodfellow and Bullough 1967, 1968). In both the elbow and the hip those areas which do not usually articulate with opposed cartilage always showed some degree of chondromalacia.

Chondromalacia is attributed to a decreased content of sulphated mucopolysaccharides in the ground substance, and this diminution can be demonstrated by a loss of basophilia in haematoxylin and eosin preparations, and more specifically by the Alcian blue technique.

If the unloaded non-contact areas were never subjected to the mechanical stress of articulation with opposed cartilage, chondromalacia at those sites might be of little significance, but this is not the case. Parts of such areas do occasionally articulate, usually at some extreme of the range of movement, and then the softened cartilage proves mechanically inadequate to support the collagen framework, its complex structure begins to break up and the next phase of degeneration is observed.

\section{FIBRILLATION}

Articular cartilage is a two-phase system structurally analogous to reinforced concrete or a motor car tyre. Fibrillation is the term applied to the exposure through loss of ground substance of the fibrous framework within. 


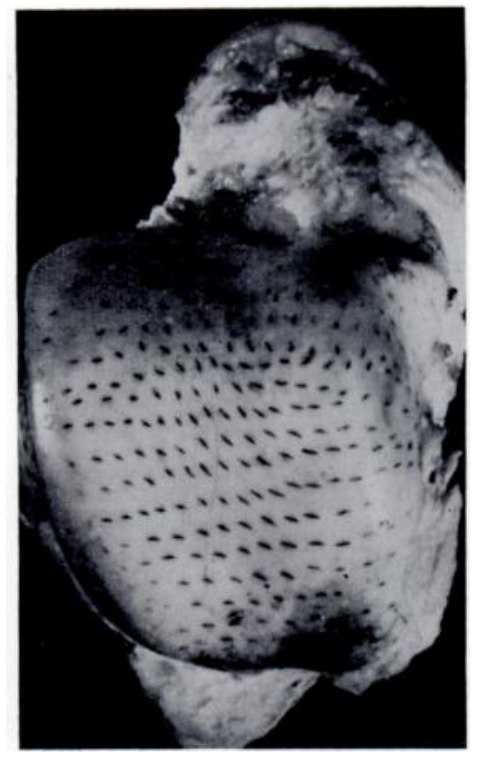

Fig. 1

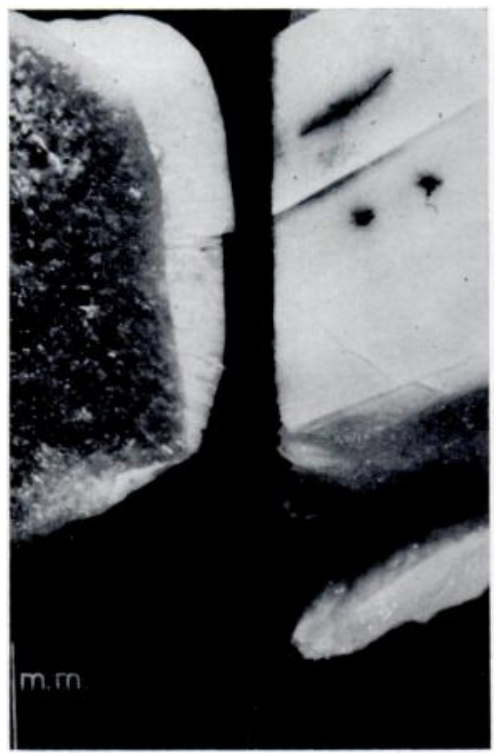

FIG. 2

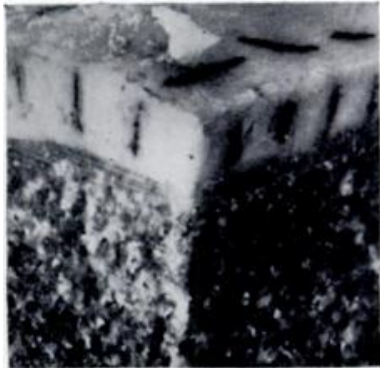

Fig. 3

Figs. 1 To 3

Figure 1-."Split line" pattern of a talus. The splits are produced by a pin, the tip of which is covered in indian ink. Figure 2 -The result of removing the outer layer of the cartilage before introducing the pin. Figure 3-" Split lines" produced on the cut edge of the cartilage.

That some fibrous system exists within normal articular cartilage is readily demonstrable by the simple expedient of pricking its surface with a pin. A split results, and if the pricking is repeated all over the surface a pattern of split lines is revealed (Fig. 1). The pattern for each joint is constant from subject to subject.

This property of articular cartilage was first demonstrated by a Swedish physician, Hultkrantz, in 1898. He believed that the split lines followed the path of joint movement. In 1925 Benninghoff showed that the cleavage lines did not in fact parallel the movement of the joint but that they connected, by the shortest route and with the least curvature, the edges of the articular surface. He proposed that the lines followed the tension trajectories set up by compression of the articular cartilage. If we accept for the moment that the fissures reflect the internal fibre arrangement of the cartilage, then we can infer that on the surface the fibres are running parallel to the surface and in the general direction of the split line. If the superficial layer of the cartilage is pared away and the exposed surface pricked, instead of fissures appearing only small round holes result (Fig. 2). If the cut edge of the cartilage is pricked a vertical split line is produced and this occurs in all planes of section (Fig. 3). These experiments indicate that in the deeper layers of the cartilage the fibres are predominantly vertical.

\section{STUDIES OF FINE STRUCTURE}

Since collagen is doubly refractile the orientation of the fibres may be studied microscopically by examining sections with polarised light (Amprino 1948). As a refinement of this method we have used a first-order red filter between the polarising and the analysing filters, permitting differentiation between fibres running at right angles to each other. We have cut our sections in strict orientation to the fissure line pattern on the surface, and it has been possible by this technique to confirm and augment the three-dimensional model suggested by macroscopic examination.

Figure 4 shows a section which has been cut parallel to a split line. On the surface there is a highly refractile zone in which the fibres run parallel to the surface (blue) and at right angles to the vertical fibres in the depths (red). Figure 5 shows a section cut across a split line. The surface layer is hardly refractile at all because most of the fibres have been transected and are viewed end on. The vertical fibres in the depths appear, of course, unaltered. 
If after demonstrating the major orientation of the collagen fibres we rotate the section on the microscope stage through an angle of 45 degrees, those fibres are no longer visible. However, at this angle to the plane of polarised light other fibres become apparent which are equally distributed between the red and the blue end of the spectrum and therefore running

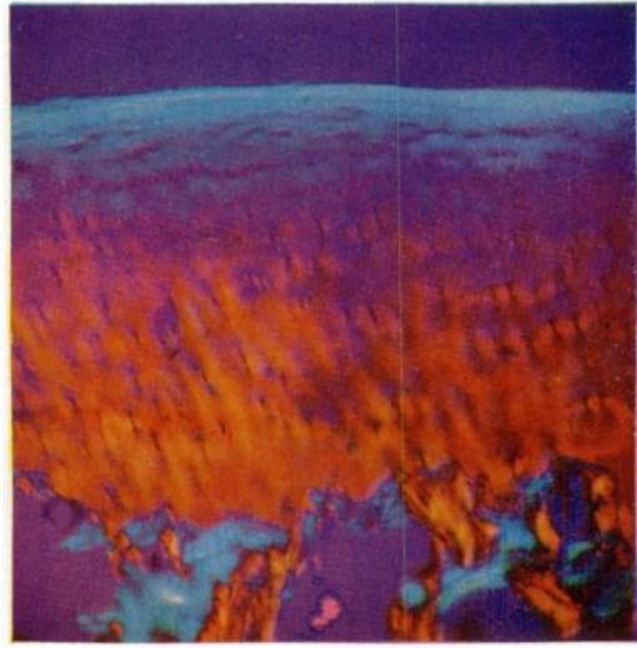

Fig. 4

Photomicrograph of a section of articular cartilage cut parallel to the split line. $(\times 20$, polarised light with first-order red filter.)

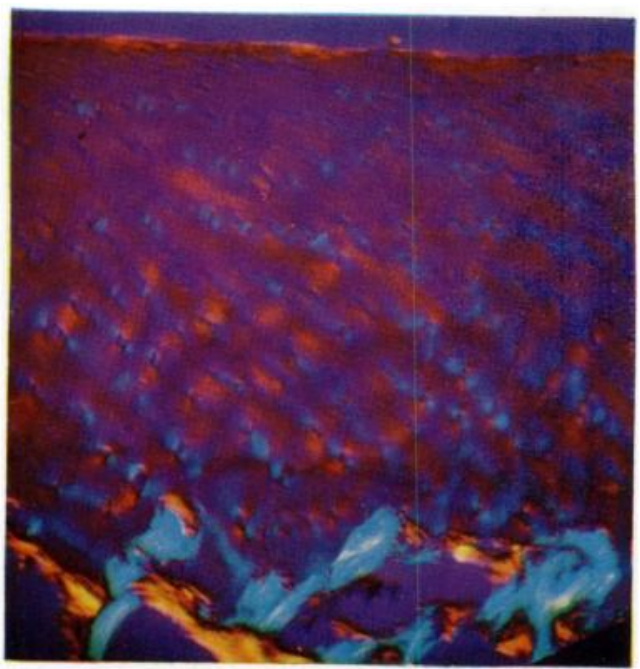

FIG. 6

As Figure 4 but with the section rotated through 45 degrees.

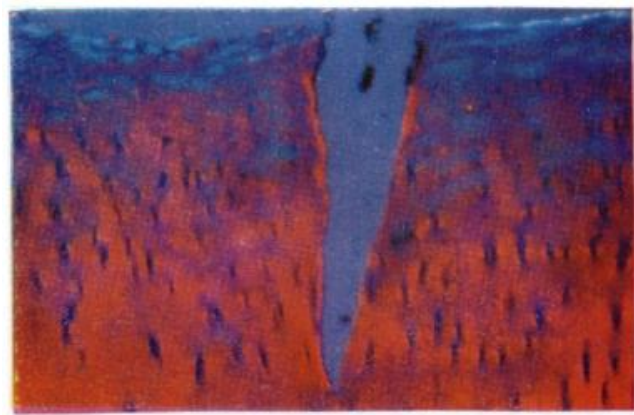

Fig. 5

Photomicrograph of a section of articular cartilage cut across a split line. $(\times 20$, polarised light with first-order red filter.)

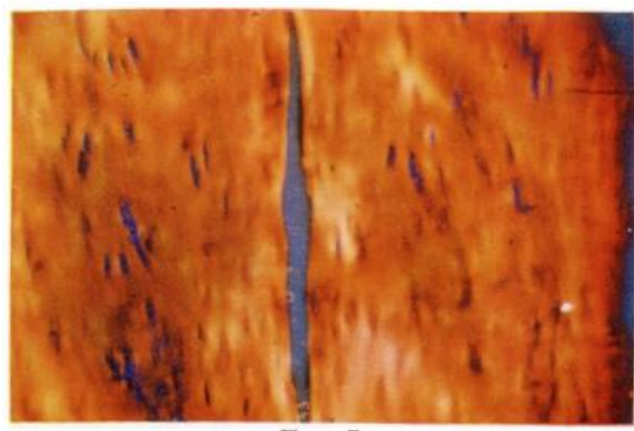

Fig. 7

Photomicrograph of a horizontal section of the surface of articular cartilage. $(\times 20$, polarised light with first-order red filter.)

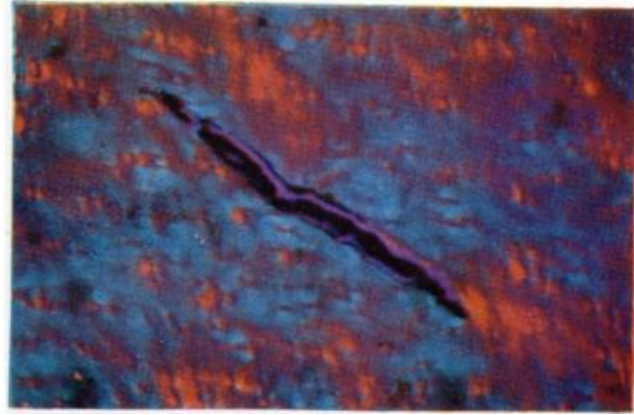

Fig. 8

As Figure 7 but with the section rotated through 45 degrees.

at right angles to each other (Fig. 6). Thus we can deduce that in addition to the principal fibre orientation there is a bracing system of fibres running generally at an angle of 45 degrees to the principal orientation.

Figure 7 shows a horizontal section of the surface to include a split line. In this plane all the visible fibres are running parallel to the split line. This is the principal orientation 
and the explanation for the direction of the split. In Figure 8 the same section is shown but the microscope stage has been rotated through an angle of 45 degrees. A bracing system of fibres running generally at an angle of 45 degrees to the principal orientation is again revealed.

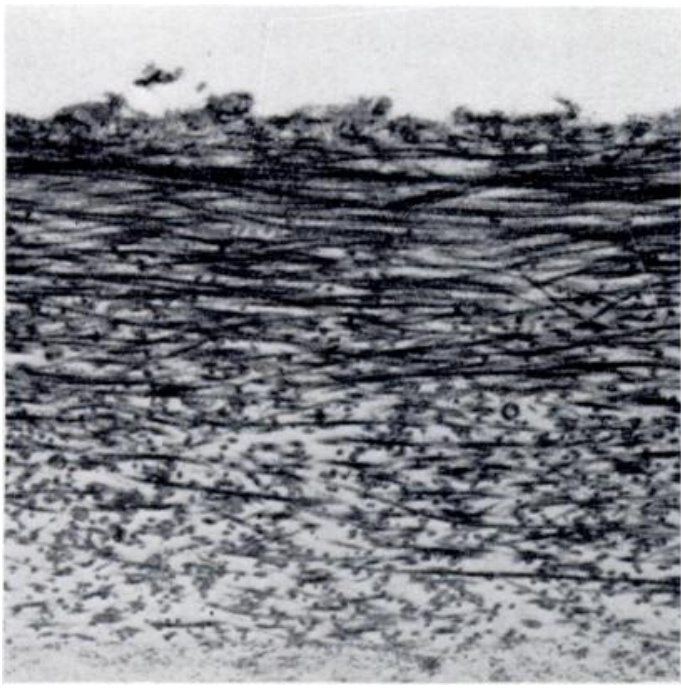

FIG. 9

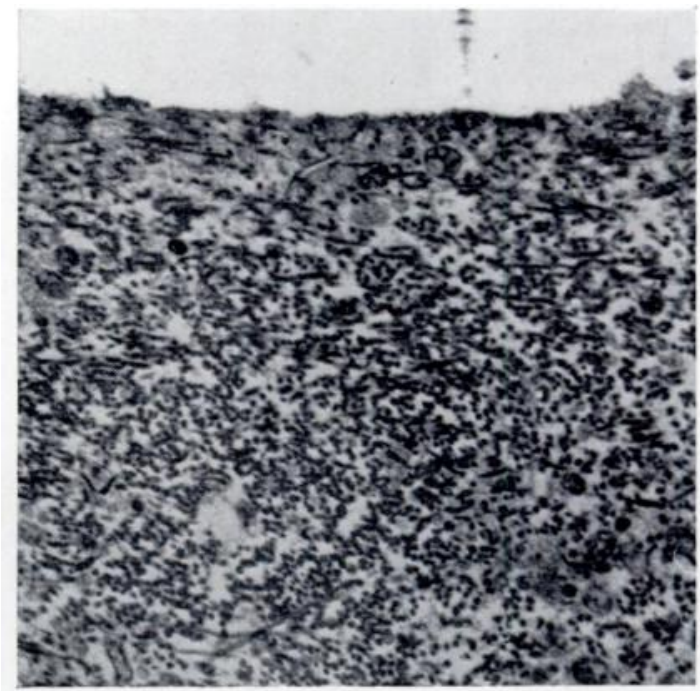

Fig. 10

Figure 9-Electron photomicrograph of ulira-thin section of articular cartilage cut parallel to the split line. Note the collagen fibrils running parallel with the surface. $(* 6,000$.) Figure 10-Electron photomicrograph of ultra-thin section of articular cartilage cut across the split line. In this plane of section most of the fibres are seen in cross-section. $(\times 6,000$.

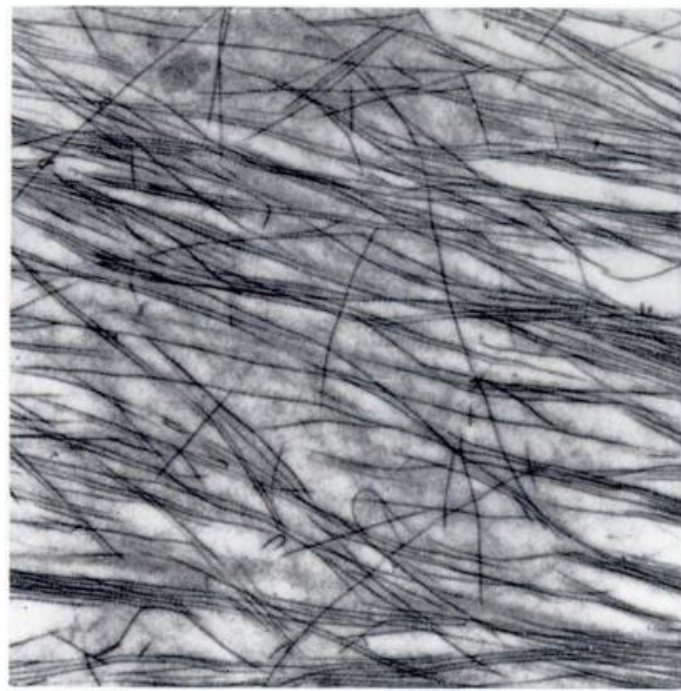

FIG. 11

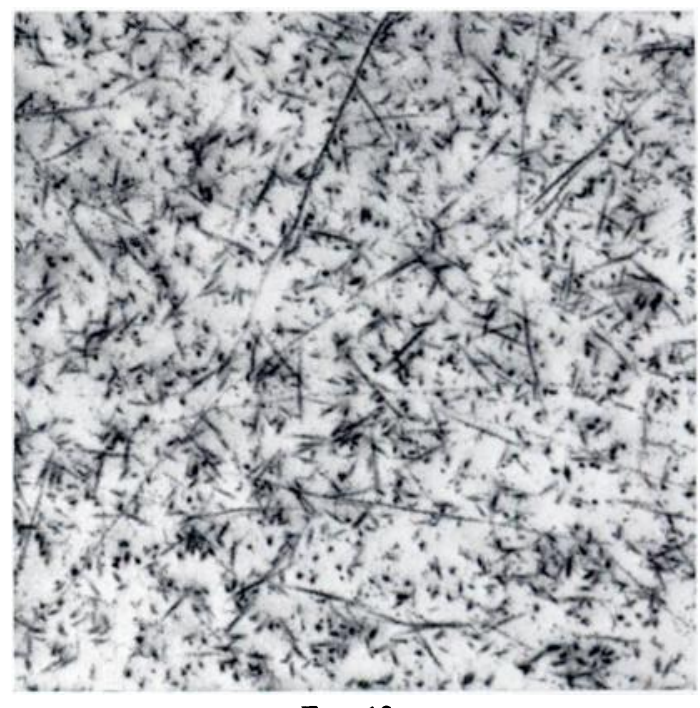

FIG. 12

Figure 11-Electron photomicrograph of ultra-thin section of articular cartilage cut immediately beneath and horizontal with the surface. Note that the general orientation of the fibres is parallel and that no fibres are seen in cross-section. $(\times 6,000$.) Figure 12 -Flectron photomicrograph of ultra-thin section of articular cartilage cut from the same block and parallel to the section illustrated in Figure 11, but at a deeper level. The vertical fibres of the articular cartilage are now seen in cross-section. $(\times 6,000$.)

Only the general alignment of the collagen fibres can be deduced from the above observations, and it is not possible by optical microscopy to visualise the individual collagen fibrils. They can, however, be demonstrated with the electron microscope. The technical 


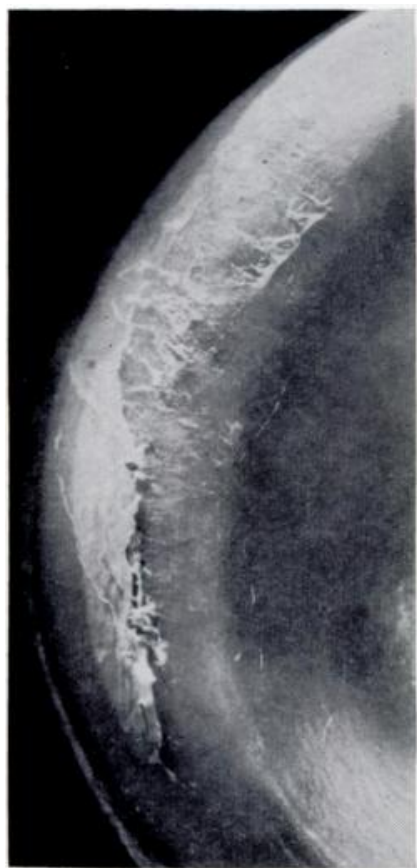

FIG. 13

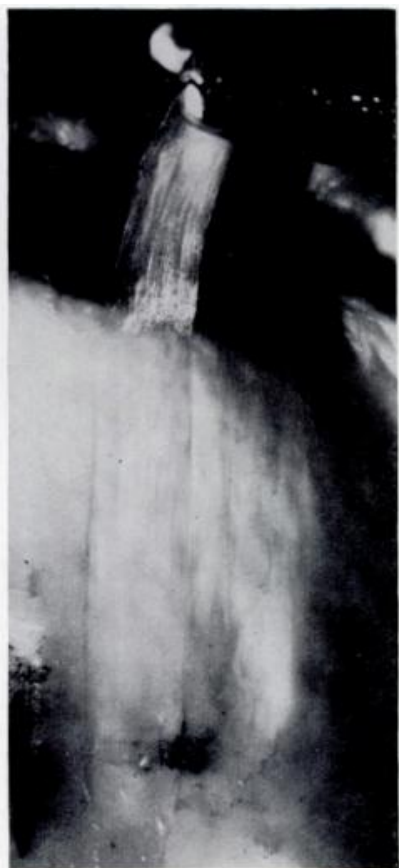

FIG. 14

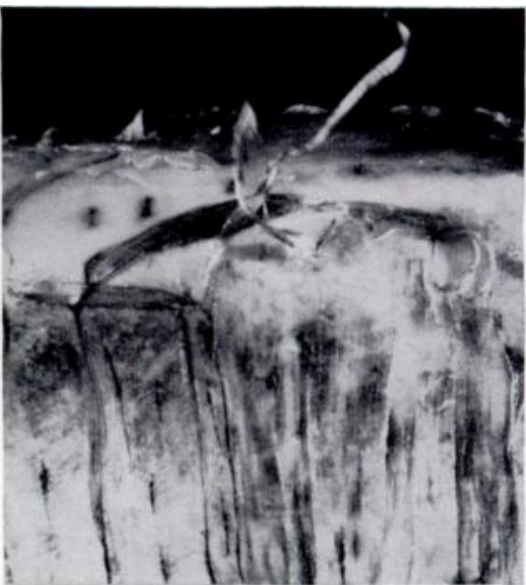

FIG. 15

FIGS. 13 To 15

Figure 13-Enlarged view of part of the radial head of an 18-year-old man showing detachment of the superficial layer of the cartilage $(\times 7)$ Figure 14-The thin surface membrane of the cartilage artificially detached. A split line is seen in the lower left corner. Figure 15-Enlarged view of part of the periphery of a patella showing detachment of the superficial layer of cartilage in the form of strands. The relationship of the detached strands to the split line pattern is demonstrated.

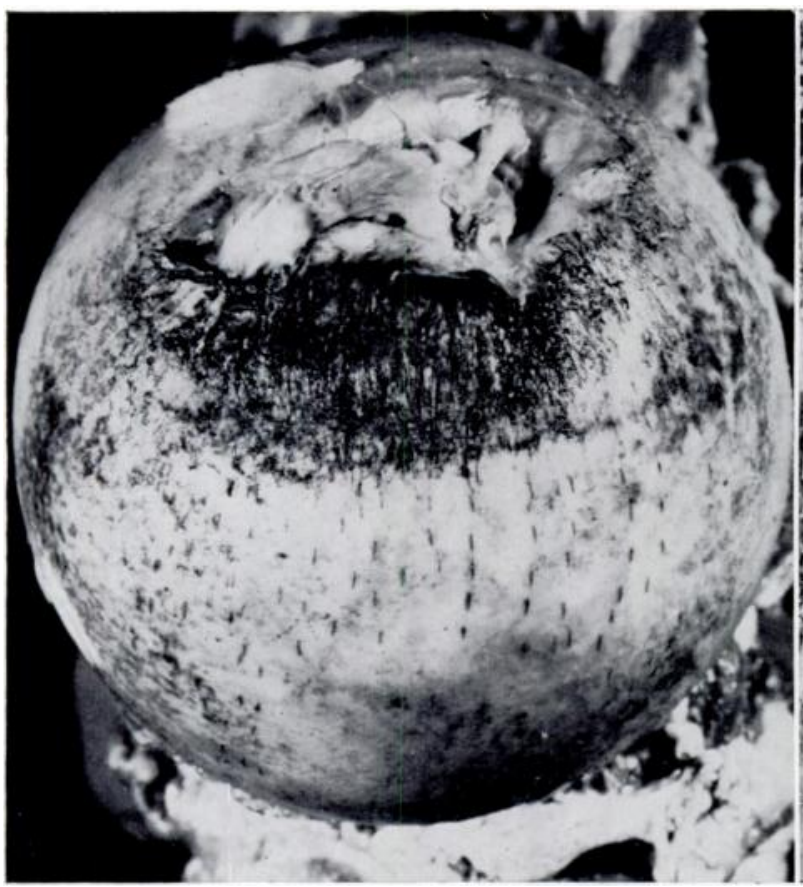

FiG. 16

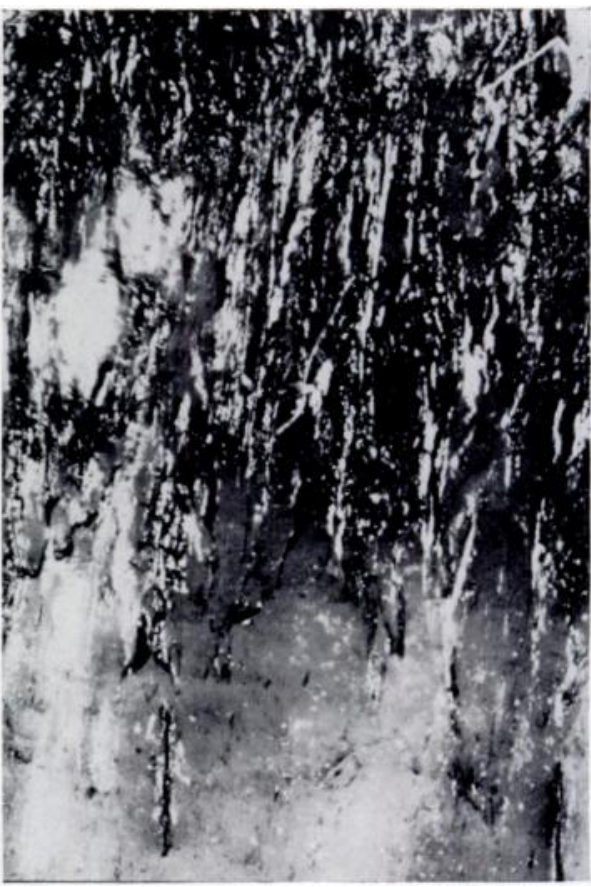

Fig. 17

Figure 16-The fibrillated cartilage around the fovea of a femoral head is demonstrated after the surface has been gently rubbed with indian ink. Figure 17-Enlarged view of Figure 16 showing the junction between the intact and fibrillated cartilage. 
problem is to orientate accurately the very small blocks from which the sections are cut. Only if one knows the exact relationship of the section to the split line pattern can one interpret the observations. The electron photomicrographs illustrated (Figs. 9 to 12) should be compared with the polarised light photographs.

The orientation of the collagen fibres, though hidden in healthy cartilage, is revealed when the cartilage degenerates. Figure 13, the rim of the radial head of an eighteen-year-old man, shows the earliest visible sign of cartilage failure. This is an area of cartilage subject to softening, and it shows blistering of the surface layer and detachment of a thin surface membrane. This membrane can be artificially detached from normal cartilage and pulled away as a sheet (Fig. 14).

Significantly, it is easier to do this if the cartilage is already malacic. Figure 15 shows a fibrillated area on the articular surface of a patella. The exposed superficial fibres are seen to be parallel with the split lines artificially produced on the adjacent cartilage. Figures 16 and 17 show the articular surface of a femoral head which has been gently rubbed with indian ink. The ink has been trapped by revealed fibres in an area of fibrillation (which is typically perifoveal) and they are seen to accord with the split line pattern in the intact cartilage. Histological sections show loss of staining in the fibrillated area from its deficiency in mucopolysaccharides.

The degenerative process described in the foregoing pages begins in areas of softened cartilage and ends with disruption of the collagen framework. To recognise that the cartilage covering a joint facet is organised as a whole is to accept that a lesion in any part may prejudice the integrity of it all. An interruption in the transmission of tension forces by a defect, even in an unloaded area, must lead to the setting up of abnormal stress patterns and predispose the adjacent loaded areas to mechanical breakdown.

\section{SUMMARY}

The collagen framework of articular cartilage is disposed, as in other connective tissues, to resist tension forces within the material. In this paper the fine structure of articular cartilage, as demonstrated by polarised light microscopy and electron microscopy, is related to the gross anatomy and to the naked eye changes of chondromalacia and fibrillation.

\section{REFERENCES}

Amprino, R. (1948): Recherches et considérations sur la structure du cartilage hyalin. Acta Anatomica, 5, 123. BenNinghoff, A. (1925a): Form und Bau der Gelenkknorpel in ihren Beziehungen zur Funktion. Erste Mitteilung Zeitschrift für Anatomie und Entwicklungsgeschichte, 76, 43.

BenNinghoff, A. (1925b): Form und Bau der Gelenkknorpel in ihren Beziehungen zur Funktion. Zweiter Teil: Zeitschrift für Zellforschung und mikroskopische Anatomie, 2, 783.

Goodfellow, J. W., and Bullough, P. G. (1967): The Pattern of Ageing of the Articular Cartilage of the Elbow Joint. Journal of Bone and Joint Surgery, 49-B, 175.

Goodfellow, J. W., and Bullough, P. G. (1968): Studies on Age Changes in the Human Hip Joint. Journal of Bone and Joint Surgery, 50-B, 222.

Hultkrantz, W. (1898): Ueber die Spaltrichtungen der Gelenkknorpel. Verhandlungen der Anatomischen Gesellschaft, Kiel, p. 248.

Kempson, G. (1967): Personal communication.

WeichSelbaum, A. (1877): Die senilen Veränderungen der Gelenke und deren Zusammenhang mit der Arthritis deformans. Sitzungsbericht der kaiserlichen Akademie der Wissenschaften, lxxv, 193. 